\title{
New insights into functional effects of the shared epitope
}

Does the shared epitope-a common five-amino-acid sequence in the $\beta 1$ subunit of the HLA-DR molecule-act as a ligand to trigger innate immune signalling? This hypothesis, put forth by Joseph Holoshitz and colleagues, could help explain the mechanism that underlies the well-established yet poorly understood relationship between $H L A D R B 1$ alleles that encode the shared epitope and susceptibility to, and severity of, rheumatoid arthritis (RA). Recent work by this group, published in The Journal of Immunology, suggests that the shared epitope has direct arthritogenic effects in RA via activation of osteoclastogenesis.

Holoshitz et al. demonstrated in vitro that treatment of mouse and human cells with a synthetic shared epitope peptide induced the differentiation of osteoclasts, and augmented the production of the proosteoclastogenic cytokines IL-6 and TNF. Furthermore, the peptide facilitated T-cellmediated effects on osteoclastogenesis, by enhancing the differentiation of $\mathrm{T}_{\mathrm{H}} 17$ cells expressing receptor activator of nuclear factor $\kappa B$ ligand (RANKL). Notably, the shared epitope effect on differentiation of mouse osteoclasts was augmented by the presence of IL-17, suggesting that the shared epitope and IL-17 act synergystically to promote bone destruction.

\section{4 .... synthetic shared epitope} peptide induced the differentiation of osteoclasts... 77

Osteoclast differentiation and function was also enhanced in bone marrow cells isolated from transgenic mice expressing genes encoding the human shared epitope, in comparison with cells from mice expressing a different HLADRB1 allele.

The researchers also examined the effects of the shared epitope in vivo, in mice with collagen-induced arthritis. Intraperitoneal injection of a shared epitope peptide led to early onset of arthritis and a transient but nonetheless significant increase in joint swelling. Articular bone erosion was also more severe in shared epitope-treated mice than in controls; consistent with this effect, osteoclasts were more abundant in synovial tissue of mice that received the peptide.

"The shared epitope ligand, devoid of any antigen presentation capabilities, was able to specifically and potently activate $\mathrm{T}_{\mathrm{H}}$ 17-cell and osteoclast differentiation in vitro and accelerated erosive arthritis in mice in vivo," says Holoshitz. "This is the first direct mechanistic insight into the role of the shared epitope in inflammatory arthritis, and the first evidence linking $\mathrm{T}_{\mathrm{H}} 17$-cell and osteoclast differentiation to the shared epitope."

\section{Sarah Onuora}

Original article Holoshitz, J. et al. An HLA-DRB1-coded signal transduction ligand facilitates inflammatory arthritis: a new mechanism of autoimmunity. J. Immunol. doi:10.4049/jimmunol.1202150 